\title{
Sporadic hand, foot, and mouth disease cases associated with non-C4 enterovirus 71 strains in Xiamen, China, from 2009 to 2018
}

\author{
Mengyuan Chen ${ }^{1}$. Shuizhen $\mathrm{He}^{2}$ - Qiang Yan ${ }^{1}$ Jianmei Zhang ${ }^{3}$. Caiyu $\mathrm{Li}^{1} \cdot$ Xiaosong Su$^{1}$ - Shiyin Zhang ${ }^{1}$ (i) . \\ Tingdong $\mathrm{Li}^{1}$. Shengxiang Ge ${ }^{1} \cdot$ Min Chen ${ }^{3}$. Jun Zhang ${ }^{1} \cdot$ Ningshao Xia $^{1,4}$
}

Received: 10 February 2021 / Accepted: 23 March 2021 / Published online: 18 May 2021

(c) The Author(s), under exclusive licence to Springer-Verlag GmbH Austria, part of Springer Nature 2021

\begin{abstract}
Enterovirus 71 (EV71) has caused large hand, foot, and mouth disease (HFMD) epidemics among young children, and EV71 infection is the leading cause of severe HFMD cases and deaths. In mainland China, the prevalence and risk factors of non-C4 EV71 strains are still unclear. In this study, we monitored non-C4 strains over a 10-year HFMD epidemiological surveillance period in Xiamen. The 5'UTR and VP1 coding region of EV71 strains were amplified by RT-nested PCR and sequenced. Thirty-two non-C4 EV71 strains were identified during 2009-2018. This study provides important information about the prevalence of EV71 in China that will be applicable for development of vaccines and diagnostic reagents as well as establishment of policies for HFMD prevention and control.
\end{abstract}

Hand, foot and mouth disease (HFMD), caused by human enteroviruses (HEVs), is a common pediatric infectious disease. In recent years, mainland China has experienced a large number of HFMD outbreaks [1, 2]. From January 2008 to June 2019, 22.54 million HFMD cases, resulting in 3,704 deaths, were reported to the national surveillance system for HFMD in China established by the Chinese Center

Handling Editor: Tim Skern.

Mengyuan Chen and Shuizhen He contributed equally.

Shiyin Zhang

zhangshiyin@xmu.edu.cn

$\triangle$ Min Chen

2049995204@qq.com

1 State Key Laboratory of Molecular Vaccinology and Molecular Diagnostics, National Institute of Diagnostics and Vaccine Development in Infectious Diseases, School of Public Health, Xiamen University, South Xiang'an Road, Xiamen 361102, China

2 Xiamen Haicang Hospital, Haiyu Road, Xiamen 361026, China

3 Xiamen Center for Disease Control and Prevention, Shengguang Road, Jimei District, Xiamen 361021, China

4 The Research Unit of Frontier Technology of Structural Vaccinology of Chinese Academy of Medical Sciences, Xiamen University, South Xiang'an Road, Xiamen 361102, China for Disease Control and Prevention (Chinese CDC) (http:// www.nhc.gov.cn/jkj/s2907/new_list.shtml?tdsourcetag=s_ pcqq_aiomsg).

The main pathogens causing HFMD in mainland China are enterovirus 71 (EV71), coxsackievirus A16 (CA16), coxsackievirus A6 (CA6), and coxsackievirus A10 (CA10). Because most of the severe cases involving nervous system syndromes and cardiopulmonary failure are caused by EV71, this virus has received the most attention by clinicians and researchers in many countries.

EV71 can be clustered into three genotypes: A, B (subgenotypes B1-B5), and C (subgenotypes C1-C5). In other parts of Asia, several subgenotypes of EV71 have been found to circulate or co-circulate in a same area [3-9], whereas in mainland China, almost all EV71 strains belong to subgenotype $\mathrm{C} 4[2,10]$, and only individual sporadic cases caused by non-C4 strains have been reported [11-13]. The prevalence and risk factors of non-C4 strains are not known. This might lead to bias in vaccine design, development of diagnostic reagents, and disease control and prevention strategies. In this study, we investigated the prevalence of non-C4 strains in a 10-year epidemic study in Xiamen, a transport hub between Southeast Asia and mainland China that may stand in the forefront of epidemic trends.

From January 2008 to September 2019, 64,309 cases, including 124 severe cases, were reported to the Xiamen CDC. During this period, there was a rising trend in HFMD cases in Xiamen (Supplementary Fig. S1). A deep 
monitoring system for HFMD-associated pathogens was implemented in Xiamen in 2009. Two sampling methods were used: from January 2009 to June 2010, samples from each individual case were collected and analyzed, whereas from July 2010 to March 2018, due to the increased number of cases, only random samples were analyzed. In total, 7,203 cases were sampled and analyzed between January 2009 and March 2018.

Samples were examined using reverse transcription nested-PCR (RT-nested PCR) as reported previously [14]. Samples from 5,315 of these cases were positive for HEVs (73.8\%). CA6 (33.63\%), EV71 (28.45\%), CA16 (23.62\%), and CA10 (4.98\%) were the primary pathogens detected (Fig. 1). Each EV71 strain was subgenotyped. The vast majority belonged to subgenotype C4. However, as VP1 analysis could only distinguish between subgenotypes $\mathrm{C} 4$ and $\mathrm{C} 5$, a small portion of strains failed to be subgenotyped.

In this study, 55 EV71 samples that could not be subgenotyped previously were analyzed. Nucleic acids were extracted using a GenMagBio Viral DNA/RNA Extraction Kit (GenMagBio, China), and 3-step RT-nested-PCR was performed using non-EV71 VP1 primers (Supplementary Table S1). The RT mixture in each tube consisted of $4 \mu \mathrm{l}$ of M-MLV buffer, $4 \mu \mathrm{l}$ of a dNTP mixture (10 mM each), 0.6 $\mu \mathrm{l}$ of specific primer $(10 \mu \mathrm{M}), 0.2 \mu \mathrm{l}$ of M-MLV Reverse Transcriptase (TaKaRa), $0.2 \mu$ l of Recombinant RNasin Ribonuclease Inhibitor (Promega), $5 \mu$ of viral RNA, and $6 \mu \mathrm{l}$ of nuclease-free water. The RT reaction was performed at $50{ }^{\circ} \mathrm{C}$ for $30 \mathrm{~min}$. The first-round PCR mixture in each tube consisted of $2 \mu \mathrm{l}$ of $10 \times$ PCR buffer, $2.4 \mu \mathrm{l}$ of dNTP mixture ( $2.5 \mathrm{mM}$ each), $0.4 \mu \mathrm{l}$ of forward primer $(10 \mu \mathrm{M})$, $0.4 \mu \mathrm{l}$ of reverse primer $(10 \mu \mathrm{M}), 0.2 \mu \mathrm{l}$ of rTaq (TaKaRa), $5 \mu \mathrm{l}$ of cDNA, and $9.6 \mu \mathrm{l}$ of nuclease-free water. The cycling conditions were as follows: initial denaturation at $95{ }^{\circ} \mathrm{C}$ for $10 \mathrm{~min}$, followed by 35 cycles of $94{ }^{\circ} \mathrm{C}$ for $40 \mathrm{~s}, 53{ }^{\circ} \mathrm{C}$ for $40 \mathrm{~s}$, and $72{ }^{\circ} \mathrm{C}$ for $1 \mathrm{~min}$, and final extension at $72{ }^{\circ} \mathrm{C}$ for $10 \mathrm{~min}$. A volume of $2 \mu \mathrm{l}$ of the first PCR product was used for the second-round PCR with $5 \mu 1$ of $10 \times$ PCR buffer, 6 $\mu l$ of dNTP mixture (2.5 mM each), $1 \mu l$ of forward primer
$(10 \mu \mathrm{M}), 1 \mu \mathrm{l}$ of reverse primer $(10 \mu \mathrm{M}), 0.5 \mu \mathrm{l}$ of rTaq (TaKaRa), and $34.5 \mu \mathrm{l}$ of nuclease-free water and using the same PCR conditions. The amplicons were purified and sequenced. All of the sequences were submitted to the GenBank sequence database (NCBI, USA) with the accession numbers MT861057-MT861109. Phylogenetic trees based on partial 5' UTR and VP1 sequences were constructed using the Jukes-Cantor algorithm and the neighbor-joining method in MEGA 7.0 software (www.megasoftware.net). As the partial VP1 sequences were from at different regions of the gene, three separate phylogenetic trees based on partial VP1 sequences were constructed.

The results indicated that these sequences belonged to non-C4 strains that are particularly uncommon in mainland China. Thirty-two non-C4 strains were identified in Xiamen (Supplementary Table S2). These cases, all of which were mild, showed clinical manifestations similar to those in patients infected with other HEVs. Based on their VP1 sequences, three $\mathrm{B} 3$ strains, $10 \mathrm{~B} 5$ strains, one $\mathrm{C} 2$ strain and one C5 strain were identified. Among the 15 strains, the 5' UTR and VP1 sequences of four strains clustered into different subgenotypes. This suggested that genotype recombination events had occurred in the region between the 5' UTR and the VP1 gene. Based on the VP1 sequences, most of the B5 strains from Xiamen were closely related to strains detected in Taiwan, while two other Xiamen B5 strains had a close relationship to strains from Southeast Asia. All Xiamen B5 strains isolated from 2010 to 2012 were closely grouped into a sublineage. The Xiamen $\mathrm{C} 2$ strain was closely related to strains from the Philippines, while the Xiamen C5 strain was related to strains from Vietnam. The Xiamen B3 strains were segregated into two sublineages.

The VP1 sequences of 17 strains failed to be amplified. Phylogenetic analysis based on 5' UTR sequences showed that one strain was genotype A, two were subgenotype B3, and three were subgenotype B5, while 11 showed similarity to orphan strains from India (Supplementary Table S2 and Fig. 2a-d). Two C4 strains were excluded based on VP1 phylogenetic analysis.
Fig. 1 Yearly distribution of detected HFMD cases (continuous line) and the pathogen spectrum of HEVs (histogram) in Xiamen, China, from January 2009 to March 2018

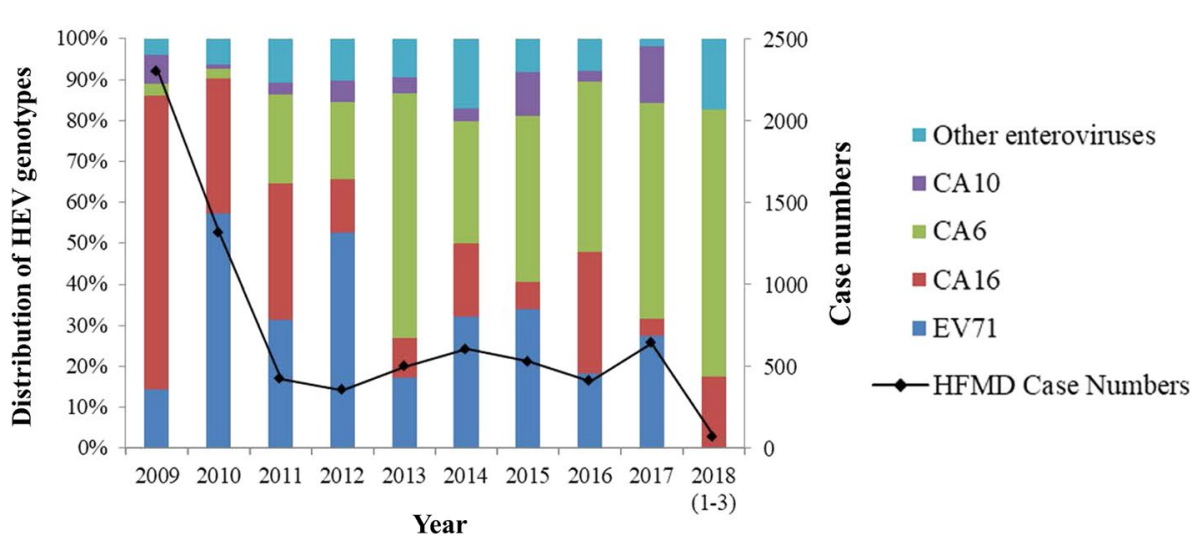


In Asia, HFMD cases are frequently associated with EV71. In Southeast Asia, including Taiwan, several EV71 subgenotypes cocirculated in the same region. However, the epidemic patterns in mainland China are entirely different. Except for isolated cases, all of the EV71 strains belong to subgenotype C4. Rare isolations of non-C4 EV71 strains have been reported in different years and areas [11-13]. We reported previously that the first imported B5 strains in Xiamen (isolated in 2009) displayed a close genetic relationship to strains present in Taiwan and Singapore in 2006-2008 [15]. Five years later, the second imported B5 strain, which shared the highest nucleotide sequence identity with strains found in Vietnam in 2011-2013, was reported in Chongqing [11]. A C2 strain was isolated in Shandong in 1998, and another was isolated in Beijing in 2015 [12, 13].

For high sensitivity, reagents used in mainland China are usually developed specifically for detection of EV71 C4 strains. The prevalence and risk factors of non-C4 strains are not clear. Furthermore, it is not known whether EV71 vaccines designed based on $\mathrm{C} 4$ strains provide cross-protection against non-C4 EV71 strains. As very few researchers have systematically studied the prevalence of non-C4 EV71 strains in mainland China, the deep surveillance of non-C4 EV71 strains described here will provide important information.

In this study, we first systematically investigated nonC4 EV71 cases in the same region of mainland China.
Thirty-two non-C4 EV71 strains, representing subgenotypes B3, B5, C2, and C3 as well as some strains resembling Indian orphan strains were identified. The non-C4 strains accounted for $2.12 \%$ of EV71 strains (32/1512, far greater than in other districts). The non-C4 strains had a close relationship to Southeast Asian strains. There might be two reasons for this. On the one hand, a deep, comprehensive, and long-term surveillance system for monitoring the pathogen spectrum of HFMD has been operating in Xiamen for 10 years. As all subgenotypes of HEVs could be identified using our system, non-C4 EV71 strains were not overlooked. On the other hand, as Xiamen is an important tourist city and a key transportation hub between mainland China and Southeast Asia, Xiamen may stand at the forefront of EV71 epidemic changes. Epidemic patterns of HEVs in Xiamen might be affected by imported HEV strains from Southeast Asia. A study in Taiwan showed that large epidemics occurred shortly after minor strains were identified a few years ago. This suggests that large epidemics caused by nonC4 EV71 strains can occur at any time, and more attention should be given to these strains.

This study provides new understanding of EV71 epidemic patterns in mainland China and also provides important information for formulating EV71 prevention and control strategies and developing vaccines and diagnostic reagents.
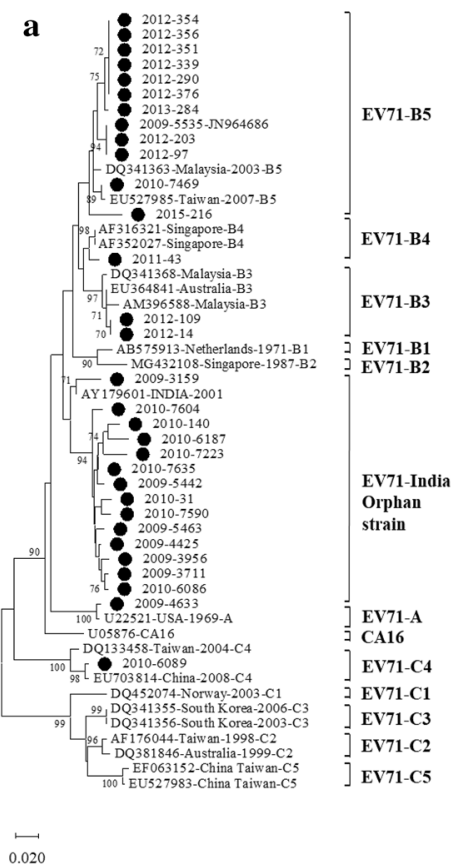

Fig. 2 Neighbor-joining phylogenetic trees constructed based on (A) partial 5' UTR sequences (corresponding to nt 206-500 of EV71 prototype strain $\mathrm{BrCr}$ U22521), (B) partial VP1 sequences (nt 26432832), (C) partial VP1 sequences (nt 2667-3130), and (D) partial

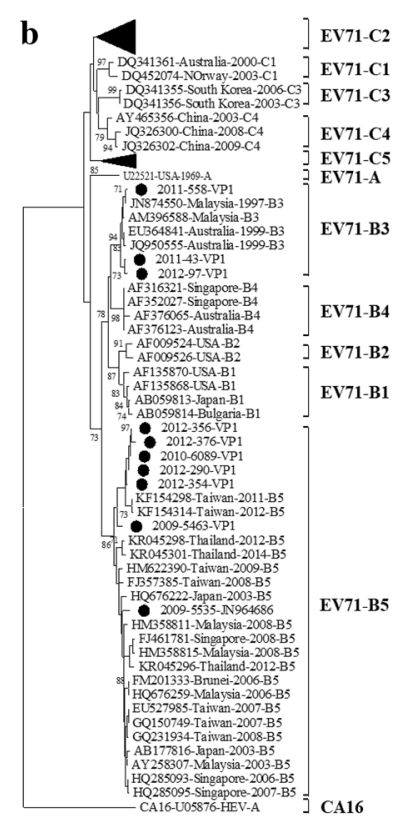

$\underset{0.05}{\varpi}$

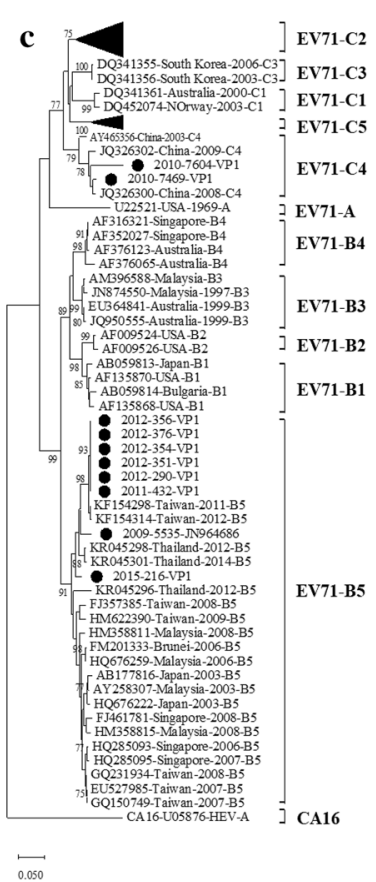

VP1 sequences (nt 2822-3033) of EV71 strains of different subgenotypes, using MEGA 7.0. Sequences from this study are indicated by the symbol ' 
Supplementary Information The online version contains supplementary material available at https://doi.org/10.1007/s00705-021-05097-9.

Author contributions The study was conceived, designed, conducted, and revised by all authors. Drafting of the manuscript and data analysis was conducted by MC, SH, SZ, and MC. All authors have read and agreed to the published version of the manuscript.

Funding This work was supported by the National Natural Science Foundation of China (31971369), the Key Program of the National Natural Science Foundation of Fujian Province (2019J02004), and the CAMS Innovation Fund for Medical Sciences (2019RU022).

\section{Declaration}

Conflict of interest The authors declare that they have no conflict of interest.

\section{References}

1. Xing W, Liao Q, Viboud C et al (2014) Hand, foot, and mouth disease in China, 2008-12: an epidemiological study. Lancet Infect Dis 14:308-318. https://doi.org/10.1016/S1473-3099(13)70342-6

2. Liu S, Pan H, Liu P, Amer S, Chan T, Zhan J, Huo X, Liu Y, Teng Z, Wang L, Zhuang H (2015) Comparative epidemiology and virology of fatal and nonfatal cases of hand, foot and mouth disease in mainland China from 2008 to 2014. Rev Med Virol 25:115-128. https://doi.org/10.1002/rmv.1827

3. Chia M, Chiang P, Chung W, Luo S, Lee M (2014) Epidemiology of enterovirus 71 infections in Taiwan. Pediatr Neonatol 55:243-249. https://doi.org/10.1016/j.pedneo.2013.07.007

4. Wu W, Kuo T, Lin Y, Huang S, Liu H, Wang J, Yzhangzh C (2013) Molecular epidemiology of enterovirus 71 infection in the central region of Taiwan from 2002 to 2012. PLoS One 8:e83711. https://doi.org/10.1371/journal.pone.0083711

5. Huang S, Hsu Y, Smith DJ et al (2009) Reemergence of enterovirus 71 in 2008 in Taiwan: dynamics of genetic and antigenic evolution from 1998 to 2008. J Clin Microbiol 47:3653-3662. https://doi.org/10.1128/JCM.00630-09

6. Wu Y, Yeo A, Phoon MC, Tan EL, Poh CL, Quak SH, Chow V (2010) The largest outbreak of hand; foot and mouth disease in
Singapore in 2008: the role of enterovirus 71 and coxsackievirus A strains. Int J Infect Dis 14:e1076-e1081. https://doi.org/10. 1016/j.ijid.2010.07.006

7. Donato C, Hoi LT, Hoa NT et al (2016) Genetic characterization of enterovirus 71 strains circulating in Vietnam in 2012. Virology 495:1-9. https://doi.org/10.1016/j.virol.2016.04.026

8. Chua KB, Kasri AR (2011) Hand foot and mouth disease due to enterovirus 71 in Malaysia. Virol Sin 26:221-228. https://doi.org/ 10.1007/s12250-011-3195-8

9. McMinn P, Lindsay K, Perera D, Chan H, Chan K, Cardosa M (2001) Phylogenetic analysis of enterovirus 71 strains isolated during linked epidemics in Malaysia, Singapore, and Western Australia. J Virol 75:7732-7738. https://doi.org/10.1128/JVI.75. 16.7732-7738.2001

10. Liu W, Wu S, Xiong Y, Li T, Wen Z, Yan M, Qin K, Liu Y, Wu J (2018) Co-circulation and genomic recombination of coxsackievirus A16 and enterovirus 71 during a large outbreak of hand, foot, and mouth disease in Central China. PLoS One 9:2337. https:// doi.org/10.1371/journal.pone.0096051

11. Yang Q, Zhang Y, Yan D, Zhu S, Wang D, Ji T, Huang W, An H, $\mathrm{Xu}$ W (2016) Isolation of an imported subgenotype B5 strain of human enterovirus A71 in Chongqing City, China, 2014. Virol J 13:115. https://doi.org/10.1186/s12985-016-0571-x

12. Tao Z, Wang H, Xu A (2012) Identification of a C2 subgenogroup strain of enterovirus 71 in a retrospective study in Shandong Province, China, from 1990 to 2010. J Clin Microbiol 50:1823-1824. https://doi.org/10.1128/JCM.06850-11

13. Li J, Li Y, Zhang S et al (2018) Analysis of an imported subgenotype C2 strain of human enterovirus 71 in Beijing, China, 2015. Front Microbiol 9:2337. https://doi.org/10.3389/fmicb.2018. 02337

14. Ge S, Yan Q, He S, Zhuang S, Niu J, Xia N (2013) Specific primer amplification of the VP1 region directed by 5' UTR sequence analysis: enterovirus testing and identification in clinical samples from hand-foot-and-mouth disease patients. J Virol Methods 193:463-469. https://doi.org/10.1016/j.jviromet.2013.06.009

15. He S, Yan Q, Xu X, Ge S, Xia N, Niu J (2013) Isolation and identification of an EV71-B5 strain. Strait J Prev Med 04:1-4

Publisher's Note Springer Nature remains neutral with regard to jurisdictional claims in published maps and institutional affiliations. 